\title{
Form-field bremsstrahlung under collision of p-branes
}

\section{Elena Melkumova*}

Department of Physics, Moscow State University, Russia

E-mail: elenamelk@mtu-net.ru

\section{Dmitry Galtsov}

Department of Physics, Moscow State University, Russia

E-mail: galtsov@phys.msu.ru

\section{Karim Salehi}

Department of Physics, Moscow State University, Russia

\begin{abstract}
We calculate classically the radiation of the antisymmetric form field generated in the collision of two non-excited membranes moving with ultrarelativistic velocities in five space-time dimensions. The interaction between branes through the form field is treated perturbatively with the deflection angle as a small parameter. Radiation arises in the second order approximation when the manifold of the minimal separation between the branes moves with the superluminal velocity. It exhibits typical Cherenkov cone features. Generalization to p-branes colliding in $D=p+3$ dimensions is straightforward.
\end{abstract}

International Europhysics Conference on High Energy Physics

July 21st - 27th 2005

Lisboa, Portugal

* Speaker. 
Recently cosmological models involving strings and branes moving in higher-dimensional space-times received a renewed attention [1]-[9]. In particular, the problem of the dimensionality of space-time can be approached within the brane gas scenario [1]-[3]. Another new suggestion is the possibility of cosmic strings which are relics of the super-strings in the early universe [4]. In both cases it seems necessary to reconsider the main mechanisms of radiation which are applicable to extended objects like strings and branes. In fact, it is well-known that dynamics of the usual cosmic strings is essentially affected by radiation processes. The radiation mechanism which has been mostly studied in the past consists in formation of excited closed loops of strings which loose their excitation energy emitting gravitons and axions. Similar mechanism applies to other branes with axions being replaced by appropriate antisymmetric forms.

Here we discuss another mechanism of radiation losses by colliding branes which may work for the unexcited branes. It is similar to bremsstrahlung of colliding charges in quantum electrodynamics. In the simplest case of two parallel strings moving in four-dimensional space-time the situation is equivalent to that of colliding charges in $1+2$ electrodynamics. More generally, strings intersecting at an angle emit radiation if the velocity of the point of their minimal separation (assuming strings to be moving in two parallel planes) has a superluminal velocity (which if infinite in the case of parallel strings). Radiation can be interpreted as the Cerenkov effect [6, 7].

Similar mechanism works for colliding p-branes (of the same type) in higher dimensions provided the brane codimension is equal to two, i.e. $D-p=3$. The Cherenkov effect arises when the hypersurface of the minimal separation of the dimension $p$ moves superluminally. We concentrate on the case of membranes in five-dimensional space time interacting via the rank three antisymmetric form potential. Gravitational interaction is neglected.

Consider two classical membranes in the flat five-dimensional (signature -++++ ) spacetime $x^{\mu}=x_{n}^{\mu}\left(\sigma^{a}\right), \mu=0,1,2,3,4$, where $n=1,2$ is the brane label, interacting with the three-form field $A_{\mu \nu \lambda}$. The world-volume of each membrane is parameterized by the internal coordinates $\sigma^{a}=(\tau, \sigma, \rho)$, and the signature is +-- .

The total action consists of the membranes and the antisymmetric form terms $S=S_{1}+S_{2}+S_{H}$, where

$$
S_{n}=-\mu_{n} \int \sqrt{-\gamma} d^{3} \sigma-\frac{4 \pi \lambda_{n}}{3 !} \int \varepsilon^{a b c} \partial_{a} x^{\mu} \partial_{b} x^{\nu} \partial_{c} x^{\lambda} A_{\mu \nu \lambda} d^{3} \sigma,
$$

where $\gamma=\operatorname{det} \gamma_{a b}$ with $\gamma_{a b}=\eta_{\mu \nu} \partial_{a} x^{\mu} \partial_{b} x^{v}$ being the induced metric, $\varepsilon^{012}=-1$, and

$$
S_{H}=\frac{1}{4 !} \int H_{\mu \nu \rho \tau} H^{\mu v \rho \tau} d^{5} x, \quad H^{\mu v \rho \tau}=4 ! \partial_{[\mu} A_{v \rho \tau]} .
$$

Here $\mu_{n}$ and $\lambda_{n}$ are the membranes tension parameters and the coupling constants. Using the worldvolume diffeomorphism invariance, one can impose three gauge conditions on the metric $\gamma_{a b}$, we choose it to be diagonal, i.e. $\dot{x}^{\mu} x_{\mu}^{\prime}=0, \dot{x}^{\mu} \bar{x}_{\mu}=0, x^{\prime \mu} \bar{x}_{\mu}=0$, where $\dot{x}^{\mu}=\partial_{\tau} x^{\mu}, x^{\prime \mu}=\partial_{\sigma} x^{\mu}, \bar{x}^{\mu}=$ $\partial_{\rho} x^{\mu}$.

The equations of motion for the membranes reads:

$$
\mu \partial_{a}\left(\gamma^{a b} \sqrt{-\gamma} \partial_{b} x^{\mu}\right)=\frac{2 \pi \lambda_{n}}{3} V^{\alpha \beta \gamma}\left(\partial^{\mu} A_{\alpha \beta \gamma}-3 \partial_{\alpha} A_{\beta \gamma}^{\mu}\right),
$$

where the world-volume form is $V^{\alpha \beta \gamma}=\varepsilon^{a b c} \partial_{a} x^{\alpha} \partial_{b} x^{\beta} \partial_{c} x^{\gamma}$. Here the three form is the sum of two contributions from membranes $A^{\mu \nu \lambda}=A_{1}^{\mu \nu \lambda}+A_{?}^{\mu \nu \lambda}$, the field equations in the Lorentz gauge 
$\partial_{\mu} A^{\mu \nu \lambda}=0$ being

$$
\square A_{n}^{\mu \nu \lambda}=4 \pi J_{n}^{\mu \nu \lambda}, \quad J_{n}^{\mu \nu \lambda}=\frac{\lambda_{n}}{2} \int V_{n}^{\mu \nu \lambda} \delta^{5}\left(x-x_{n}(\sigma)\right) d^{3} \sigma
$$

Our calculation follows the approach of [7]. We solve the branes equations of motion and the wave equation for the form field iteratively in terms of the coupling constant $\lambda$. In the zero order approximation the branes are flat and moving freely:

$$
{ }_{0} x_{n}^{\mu}=d_{n}^{\mu}+u_{n}^{\mu} \tau^{n}+\Sigma_{n}^{\mu} \sigma^{n}+\Xi_{n}^{\mu} \rho^{n}
$$

with the impact parameter $d^{\mu}=d_{2}^{\mu}-d_{1}^{\mu}$, the 5-velocities $u_{n}^{\mu}$ and the orientation vectors $\Sigma_{n}^{\mu}, \Xi_{n}^{\mu}$, with $\tau^{n}, \sigma^{n}, \rho^{n}$ being the world-volume parameters on the branes. The first brane is rest $u_{1}^{\mu}=$ $(1,0,0,0,0)$ and assumed to lie in the $3-4$ plane: $\Sigma_{1}^{\mu}=(0,0,0,1,0), \quad \Xi_{1}^{\mu}=(0,0,0,0,1)$. The second brane is inclined at the angle $\alpha$ to the first and moves with the velocity $v$ orthogonal to the brane itself:

$$
\begin{aligned}
u_{2}^{\mu} & =\gamma(1,0,-v \cos \alpha, v \sin \alpha \cos \beta, v \sin \alpha \sin \beta) \\
\Sigma_{2}^{\mu} & =(0,0, \sin \alpha, \cos \alpha \cos \beta, \cos \alpha \sin \beta) \\
\Xi_{2}^{\mu} & =(0,0,0,-\sin \beta, \cos \beta)
\end{aligned}
$$

where $\gamma=\left(1-v^{2}\right)^{-\frac{1}{2}}$ and $v=\left(1-\left(u_{1} u_{2}\right)^{-2}\right)^{\frac{1}{2}}$. If $d^{\mu} \neq 0$, the branes do not intersect physically, while the line of their minimal separation moves with the velocity

$$
V^{\mu}=\frac{v}{\sin \alpha}(0,0,0, \cos \beta, \sin \beta) .
$$

Substituting these parametrization into (4) one finds the first order form fields ${ }_{1} A_{n}^{\mu \nu \lambda}$ which describes mutual interaction between the branes. This interaction gives rise to deformations of the branes located mostly around the line of their minimal separation. Deformations serve as a source of the second order fields ${ }_{2} A_{n}^{\mu v \lambda}$ which contain the radiative parts. Radiation arises if the effective source moves superluminally, i.e. $\sin \alpha<v$. It has typical Cherenkov angular distribution determined by the equation $\omega=\mathbf{k} \mathbf{V}$, which fixes two components of the four-dimensional wave-vector $\mathbf{k}$ parallel to the brane at rest: $k^{3} v=\omega \sin \alpha \cos \beta, k^{4} v=\omega \sin \alpha \sin \beta$.

The radiation power can be computed as the rate of reaction produced by the half sum of the retarded and advanced fields upon the source and presented in the standard form [7]

$$
P^{0}=\left.\left.\frac{1}{6 \pi^{2}} \int k^{0} \varepsilon\left(k^{0}\right)\right|_{1} J_{\alpha \beta \gamma}(k)\right|^{2} \delta\left(k^{2}\right) d^{5} k,
$$

where $\varepsilon$ is the sign function and the subscript 1 indicates that the current generating the radiation field results from the first iteration order in the branes equations of motion. A closed form expression for the bremsstrahlung from the collision of two branes can be obtained analytically in the case of the ultrarelativistic collision with the Lorentz factor $\gamma=\left(1-v^{2}\right)^{-1 / 2} \gg 1$. The final formula for the spectral-angular distribution of radiated energy per unit area of the radiated brane reads:

$$
\frac{1}{S} \frac{d P^{0}}{d \omega d \chi}=\frac{64 \pi^{4} \lambda^{6} \kappa^{2}}{\mu^{2} \omega} \frac{\left(1-\kappa^{2} \chi^{2}\right)^{2}}{\left(1+\kappa^{2} \chi^{2}\right)^{4}} \exp \left(-\frac{\omega d\left(1+\kappa^{2} \chi^{2}\right)}{\gamma \kappa}\right),
$$


where $\kappa=\gamma \cos \alpha$ and $\chi=\frac{\pi}{2}+\phi$ and $l_{1}$ and $l_{2}$ are the length and $S$ is the normalization area. The spectrum has an infrared divergence due to the logarithmic dependence of the interaction potential between branes with the distance. Choosing as an infrared cutoff an inverse length parameter $\Delta$ and integrating and over frequencies we obtain the angular distribution of the total radiation

$$
\frac{1}{S} \frac{d P^{0}}{d \chi}=\frac{64 \pi^{4} f^{6} \kappa^{2}}{\mu^{2}} \frac{\left(1-\kappa^{2} \beta^{2}\right)^{2}}{\left(1+\kappa^{2} \beta^{2}\right)^{4}} \operatorname{Ei}\left(1, \frac{d\left(1+\beta^{2} \gamma \kappa\right)}{\gamma \kappa \Delta}\right) .
$$

Since the integral exponential function decays exponentially for the values of the argument, the total radiation is peaked around the direction $\chi=0(\varphi=-\pi / 2)$ within the angle $\chi \lesssim \frac{1}{\sqrt{\gamma \kappa}}$. This is conformal with the Cerenkov nature of the effect.

Similar considerations apply to radiation of p-branes colliding at a superluminal angle in $D=$ $p+3$ dimensions (i.e. in the case of branes of spatial codimension two). In this case the manifold of the minimal separation has the spatial dimension $D-4$, and the resulting expression for the radiated power differs only by the normalization brane volume factor:

$$
\frac{1}{\operatorname{Vol}_{D-3}} \frac{d P^{0}}{d \omega d \chi}=\frac{64 \pi^{4} \lambda_{D}^{6} \kappa^{2}}{\mu_{D}^{2} \omega} \frac{\left(1-\kappa^{2} \chi^{2}\right)^{2}}{\left(1+\kappa^{2} \chi^{2}\right)^{4}} \exp \left(-\frac{\omega d\left(1+\kappa^{2} \chi^{2}\right)}{\gamma \kappa}\right) .
$$

Other mechanisms of radiation from p-branes were considered in $[8,9,10]$. The work was supported in part by the RFBR grant 02-04-16949.

\section{References}

[1] R. Durer, M. Kunz, M. Sakellariadou, Why do we live in 3+1 dimensions? Phys. Lett. B614 (2005) 125, [hep-th/0501163].

[2] S. Alexander, R. Brandenberger and D. Easson, Brane gas in early univers, Phys. Rev. D52 (2000) 103509, [hep-th/0005212].

[3] D.A. Easson, Brane gas cosmology and loitering, International Workshop on Particle Physics and the Early Universe (COSMO-01), Rovaniemi, Finland, 30 Aug - 4 Sep 2001, (MCGILL-01-24, Nov. 2001), [hep-th/0111055].

[4] J. Polchinski, Cosmic string revisited, AIP.Conf.Proc 743 (2004) 331; Int. J. Mod. Phys. A20 (2004) 3413, [hep-th/0410082].

[5] A.C. Davis and T.W.B. Kibble, Fundamental cosmic strings, Contemp. Phys. 46 (2005) 313, [hep-th/0505050].

[6] D.V. Gal'tsov, D.V. Grats, and P.S. Letelier, Post-linear formalism for gravitating strings: crossed straight strings collision, Ann.of phys. 224 (1993) 90.

[7] D.V. Gal'tsov, E.Yu. Melkumova and R. Kerner, Axion bremsstrahlung from collisions of global strings, Phys. Rev. D70 (2004) 045009, [astro-ph / 0310718 ].

[8] M. Abou-Zeid, M.S. Costa, Radiation from accelerated brane, Phys. Rev. D61 (2000) 106007 , [hep-th/9909148].

[9] D.V. Galtsov and E.Yu. Melkumova, Gravitational and dilaton radiation from relativistic membranes, Phys. Rev. D63 (2001) 064025, [gr-qc/ 0006087 ].

[10] T. Harmark, and K.G. Savvidy Ramond-Ramond field radiation from rotating ellipsoidal membrans, Nucl. Phys. $\mathbf{B 5 8 5}$ (2000) 567, [hep-th/ 0002157$].$ 